\title{
HISTEROSCOPIA OFICINAL: ESTADO DEL ARTE Y REVISIÓN DE LA LITERATURA
}

\author{
Néstor Wandurraga MD*, Henry Octavio Rodríguez MD**, Ángel David Miranda MD***, \\ Carlos Giovani Castro MD***, Rodrigo Ernesto Rodríguez MD**
}

\begin{abstract}
Resumen
Objetivo: describir el estado del arte de la histeroscopia oficinal y revisar la evidencia en relación con la aceptación, tolerancia y beneficios. Metodología: búsqueda en las bases de datos PUBMED, COCHRANE, LILACS utilizando los términos Office Hysteroscopy en inglés y español publicados en los últimos diez años, que estuvieran disponibles en texto completo, además se buscó en la base de datos EMBASE con límites adicionales de metanálisis o revisión sistemática. Se incluyeron algunas guías de manejo y otros artículos relevantes a partir de las referencias bibliográficas de las búsquedas iniciales. Conclusiones: se considera un procedimiento eficaz para el diagnóstico y tratamiento de la patología intrauterina, es mínimamente invasiva y de alto grado de seguridad con una técnica adecuada. Ha sido aceptada y bien tolerada por las pacientes, incluso sin requerir anestesia.
\end{abstract}

Palabras clave: patología intrauterina, histeroscopia oficinal, pacientes ambulatorios.

Abreviaturas: HUA, hemorragia uterina anormal; HO, histeroscopia oficinal.

\section{HISTERESCOPIA OFICINAL}

\begin{abstract}
Objective: to describe the state-of-the-art and review the evidence for acceptance, tolerance and benefits of office hysteroscopy. Methodology: a search in PUBMED, COCHRANE, LILACS data bases using the terms Office Hysteroscopy in English and Spanish, published in the last 10 years, available in full text. Additionally, a search in EMBASE data bases with additional limitations of meta-analysis or systematic review. Some management guidelines and other relevant articles from the bibliographic references of the initial searches were included. Conclusions: it is considered an efficient procedure used to diagnose and treat intrauterine pathology. It is a minimally invasive and very safe intervention when performed using an adequate technique. It has been accepted and well tolerated by patients, even when no anesthesia is required.
\end{abstract}

Key words: intrauterine pathology, office hysteroscopy, outpatients

Fecha recibido: mayo 23 de 2014 - Fecha aceptado: agosto II de 2014

* Residente de segunda especialidad en Cirugía Endoscópica Ginecológica. Fundación Universitaria de Ciencias de la Salud. Bogotá DC, Colombia.

\footnotetext{
** Instructor Asistente Cirugía Endoscópica Ginecológica. Fundación Universitaria de Ciencias de la Salud. Bogotá DC, Colombia.

*** Profesor Asistente de Cirugía Endoscópica Ginecológica. Fundación Universitaria de Ciencias de la Salud. Bogotá DC, Colombia.
} 


\section{Introducción}

La hemorragia uterina anormal (HUA) es uno de los problemas de salud que más enfrentan las mujeres, pudiendo afectar alrededor del $20 \%$ en la edad reproductiva, considerándola responsable de casi dos tercios de todas las histerectomías. ${ }^{1}$ La HUA hasta hace muy poco había sido estudiada mediante dilatación y curetaje, sin embargo existe una tendencia hacia el uso de técnicas mínimamente invasivas como ecografía, biopsia endometrial ambulatoria e histeroscopia, siendo esta última de especial relevancia por cuanto nos permite ver y tratar patologías endocervicales y endometriales ${ }^{2}$, sobre todo las que guardan relación con la primera parte del acrónimo PALM-COEIN. Este resume las posibles causas de HUA en mujeres no grávidas en edad reproductiva, del sistema de clasificación desarrollado hace poco por la Federación Internacional de Ginecología y Obstetricia, en donde las causas estructurales que se pueden observar con técnicas de imagen y/o por histopatología están en PALM (Polyp, Adenomyosis, Leiomyoma, Malignancy \& hyperplasia) y las causas no estructurales relacionadas con entidades no definidas por estudios de formación de imagen o por la histopatología corresponden a COEIN (Coagulopathy, Ovulatory dysfunction, Endometrial, Iatrogenic, Not yet classified). ${ }^{3}$ Los hallazgos histeroscópicos anormales incluyen alteraciones de los vasos o capilares sanguíneos, pólipos, leiomiomas submucosos, sinequias intrauterinas, cuerpos extraños, productos de la concepción retenidos y anomalías del desarrollo mülleriano entre otros. ${ }^{2}$

La histeroscopia puede ser diagnóstica o quirúrgica; la primera permite visualizar el canal cervical, la cavidad endometrial y el ostium de las trompas de Falopio. En caso que se requiera puede ser quirúrgica, utilizando finos instrumentos de disección y corte mecánico, así como instrumentos para electrocirugía o de rayos láser. ${ }^{2}$ Las complicaciones más frecuentes son hemorragia, perforación uterina y laceración cervical. ${ }^{1,2}$ Las perforaciones uterinas en la línea media rara vez producen morbilidad significativa, salvo en los casos en que se utilice electrocirugía o laser; las laterales en cambio pueden ocasionar hematomas retroperitoneales y las perforaciones cervicales pueden dar lugar a hemorragias significativas. La laparoscopia es útil para descartar una eventual lesión intestinal o daño de la vejiga, así como para determinar su extensión. ${ }^{2}$ Teniendo en cuenta que la HO en nuestro medio aún no ha sido plenamente aceptada ni se realiza de manera masiva, la presente investigación pretende definir el estado del arte y evaluar lo descrito en cuanto a las ventajas que pudiese tener como método que permite establecer un diagnóstico visual y la toma simultanea de biopsia dirigida a menores costos.

\section{Métodos}

Se realizaron búsquedas de la literatura en las bases de datos PUBMED, COCHRANE y LILACS utilizando los términos Office hysteroscopy, limitando la búsqueda al idioma inglés y en tiempo a los últimos diez años, que estuvieran disponibles en texto completo. Además se buscó en la base de datos EMBASE con límites adicionales de metanálisis o revisión sistemática. Se incluyeron algunas guías de manejo y otros artículos relevantes obtenidos a partir de las referencias bibliográficas de las búsquedas iniciales. En total fueron seleccionados 46 artículos de los cuales seis eran revisiones sistemáticas o metanálisis, 14 ensayos clínicos aleatorios (ECA), un estudio de cohorte prospectiva, 14 estudios observacionales descriptivos, 3 guías de manejo y 8 revisiones no sistemáticas.

\section{Generalidades y recomendaciones técnicas}

La historia y el desarrollo de la histeroscopia data desde hace más de dos siglos. Los intentos de hacerla consistieron en instrumentos que podrían ser insertados dentro del útero y tratar de reflejar la luz externa para visualización de la cavidad. ${ }^{4}$ Bozzini (1773-1809), médico alemán ideó el instrumento que consistía en un tubo hueco equipado con un espejo cóncavo que trasmitía la luz de una vela, permitiendo de este modo la visualización de algunas cavidades del cuerpo como la bucal, nasal, las orejas, la vagina, el cérvix uterino y el útero, así como la uretra, la vejiga urinaria y el recto. ${ }^{4}$ A Pantaleoni se le atribuye haber realizado la primera intervención quirúrgica por histeroscopia 
en 1869 en una mujer posmenopáusica con HUA hallando pólipos dentro del útero, los cuales cauterizó con nitrato de plata bajo visión. Nitze en 1879 desarrolló un sistema de cistoscopia por medio de un asa de platino incandescente que permitía la iluminación distal. ${ }^{4}$ Pasaron alrededor de 100 años para ver el desarrollo y evolución de la histeroscopia favorecida por avances técnicos de diseño de los sistemas ópticos, que permiten una mejor observación de la cavidad uterina. ${ }^{5}$ T. Mohri en 1964 mediante la introducción de la fibra óptica y de histeroscopios flexibles logró la visualización intrauterina de los embarazos iniciales. ${ }^{4}$ Hoy en día hay modelos flexibles y rígidos, de flujo continuo con dos canales para permitir que el medio de distensión fluya hacia la cavidad uterina mientras que el fluido de desecho sale por otro ducto para ser recopilado a través de un sistema extractor, además de un canal operatorio para la toma de biopsia endometrial o cirugía intrauterina., ${ }^{2,6}$

Como medios de distensión se han usado líquidos y gaseosos. En una revisión sistemática no se encontraron diferencias estadísticas significativas en las puntuaciones de dolor cuando se utilizaron dióxido de carbono o solución salina normal. ${ }^{7}$ Sin embargo en el meta-análisis de Craciunas se analizaron sistemáticamente diez ECA con 1.839 mujeres (905 en el grupo de $\mathrm{CO} 2,934$ en el grupo de SSN), encontrándose que con el primero se presentaba más dolor y efectos secundarios, y por tanto menos satisfacción por las pacientes, además de una baja calidad visual durante el examen y una alta duración de la intervención quirúrgica. ${ }^{8}$ Se ha recomendado cuantificar los líquidos infundidos y drenados con el fin de estimar el déficit, siendo el límite máximo aceptado de $2.500 \mathrm{ml}$ para pacientes sanos, siempre y cuando se utilicen soluciones isotónicas, mientras que en edad avanzada o con condiciones comórbidas, se acepta un máximo de 750 $\mathrm{ml}$ de déficit. ${ }^{9}$ En caso de utilizar soluciones hipotónicas como la glicina al $1,5 \%$, se ha recomendado un límite de déficit de fluidos de $1.000 \mathrm{cc}$ para pacientes sanas. ${ }^{9}$ La aplicación preoperatoria de dilatadores osmóticos como la laminaria o el pre-tratamiento con misoprostol se han descrito para facilitar la dilatación cervical, disminuir las dificultades en la entrada y minimizar el dolor. ${ }^{10}$ También se ha reportado el empleo de otros fármacos como el mifepristone, el cual no ha mostrado una verdadera utilidad. ${ }^{11}$ Los diferentes estudios han producido resultados contradictorios en cuanto al real beneficio del misoprostol sobre la dilatación cervical y/o el dolor durante el procedimiento. ${ }^{10-14}$ Cooper y col. en su revisión sistemática concluyen que no hay evidencia para recomendar la administración sistemática de mifepristone o misoprostol antes de la histeroscopia ambulatoria y que la preparación cervical con prostaglandinas vaginales solo debería considerarse en mujeres posmenopáusicas cuando se utilicen sistemas histeroscópicos mayores de $5 \mathrm{~mm}$ de diámetro. ${ }^{12}$

En mujeres premenopáusicas con ciclos menstruales regulares, el momento ideal para la histeroscopia diagnóstica es durante la fase folicular temprana, inmediatamente después de la menstruación debido a que durante la fase secretora del ciclo el endometrio es grueso y podría imitar pólipos. ${ }^{2}$

\section{La histeroscopia oficinal}

Es factible gracias al menor diámetro de los telescopios, mejores protocolos de analgesia y menor costo comparada con los procedimientos de quirófano. ${ }^{6,15}$ Con un abordaje vaginal se ha descrito buena tolerancia, seguridad y con bajo riesgo de complicaciones a corto y largo plazo. ${ }^{16}$ Según el estudio de van Dongen $\mathrm{H}$, la histeroscopia de consultorio fue preferida más que la histerosonografía a pesar de que esta última resultara menos dolorosa. ${ }^{17}$ Con un abordaje vaginal (sin espéculo ni tenáculo) se ha descrito mejor tolerancia que el abordaje tradicional pinzando el cérvix ${ }^{18}$, pudiéndose realizar incluso procedimientos de esterilización tubárica histeroscópica en menos de diez minutos de manera ambulatoria en un consultorio y sin anestesia general, de manera más sencilla que la ligadura de trompas por laparoscopia. ${ }^{19}$ Esta vía vaginal propuesta por Bettocchi consiste en un acceso directo a través de la vagina conocida como abordaje por vaginoscopia, sin requerir espéculo vaginal ni tenáculo para tracción del cérvix, evitando así el dolor provocado por la excesiva manipulación de la paciente. ${ }^{5}$ Como norma no se requiere profilaxis antibiótica para la $\mathrm{HO}$ ambulatoria y no suele recomendarse..$^{20,21}$ Antes 
de realizar la histeroscopia el cirujano debe realizar el examen pélvico para determinar la posición uterina. Para ello solo se requiere el histeroscopio con una funda exterior menor de $5 \mathrm{~mm}$ de diámetro, un sistema de infusión del medio de distensión y una fuente de luz. ${ }^{2}$ Filiz y col. demostraron superioridad de la HO frente a la convencional (de quirófano) en cuanto a mejor nivel de satisfacción y un costo 3,6 veces menor, por lo cual lo califican como un procedimiento seguro y satisfactorio para la paciente, que proporciona una opción rápida de ver y tratar a bajo costo, de manera ambulatoria y sin necesidad de anestesia general, por lo cual la recomiendan como primera línea diagnóstica y con la opción de ser terapéutica. ${ }^{15}$

Indicaciones: la $\mathrm{HO}$ está indicada en el estudio de HUA, que puede estar causada por pólipos endometriales o miomas submucosos, por anormalidades en estudios de imagen como el ultrasonido transvaginal o la histerosalpingografía, ante la sospecha de tabiques uterinos o lesiones focalizadas y dispositivos intrauterinos (DIU) perdidos y detectados por ultrasonido o rayos $\mathrm{X}$. En el consultorio también se pueden realizar intervenciones quirúrgicas menores como la toma de biopsias dirigidas, extracción de DIU, lisis de adherencias y resección de pequeños pólipos menores de $2 \mathrm{~cm}$ y/o miomas submucosos menores de $3 \mathrm{~cm} .{ }^{22}$

Una de las indicaciones de la $\mathrm{HO}$ es la extracción de cuerpos extraños o dispositivos intrauterinos, que puede ser realizada con facilidad en el consultorio disponiendo para ello de un histeroscopio con camisa de flujo continuo y canal operatorio, una fuente de luz adecuada y suero fisiológico para distender la cavidad endometrial. No se requiere preparación previa, dilatación cervical, ni anestesia. ${ }^{22}$

Las principales barreras para el éxito son dolor, estenosis cervical y mala visualización del cuello uterino, por lo cual es muy importante la adecuada selección de la paciente y el asesoramiento preoperatorio de la misma, evitándose en la medida de lo posible cuando hay comorbilidades, alto nivel de ansiedad, limitaciones en la movilidad o patología uterina significativa que requiera procedimientos quirúrgicos complejos. ${ }^{2}$ Cuando hay historia personal de dismenorrea severa parece existir una menor tolerancia al dolor durante la $\mathrm{HO}$, por lo que algunos recomiendan el uso de analgesia preventiva para su realización. ${ }^{23} \mathrm{La}$ administración oral o intravaginal previa de misoprostol ${ }^{10,14}$, de antiinflamatorios no esteroideos (AINEs) ${ }^{24} \mathrm{o}$ ansiolíticos, el bloqueo anestésico paracervical ${ }^{25}$, la utilización de histeroscopios delgados (menores de $5 \mathrm{~mm}$ de diámetro) o flexibles y personal auxiliar con adecuado entrenamiento, puede facilitar el procedimiento. ${ }^{2,10}$ Sharma y col. compararon el uso preoperatorio de un antiespasmódico conocido como drotaverina combinado con ácido mefenámico por vía oral, versus bloqueo paracervical o la sedación con diazepam por vía intravenosa, encontrando mejor tolerancia al dolor usando antiespasmódico combinado con el AINE que con el bloqueo paracervical o la sedación con la benzodiacepina. ${ }^{24}$ Los resultados de disminución del dolor con el uso preoperatorio de lidocaina en gel son contradictorios. ${ }^{26,27}$ En el metaanálisis de Ahmad hay evidencia del beneficio en el uso de anestésicos locales y concluyen que pueden considerarse al realizar la histeroscopia en mujeres después de la menopausia para reducir la tasa de fracaso debido al dolor. ${ }^{28}$ Bastu y col. realizaron un estudio doble ciego, aleatorio y controlado con sesenta mujeres infértiles que requerían $\mathrm{HO}$, demostrando que con el misoprostol preoperatorio se lograba una entrada cervical más fácil, breve duración del procedimiento y menor dolor, aunque el aumento de la dosis de misoprostol de $200 \mathrm{mcg}$ a 400 mcg no mejoró el efecto de la dilatación cervical. ${ }^{10}$ Otro estudio similar reveló la superioridad del misoprostol frente a la lidocaína en spray para el manejo del dolor, ${ }^{14}$ pero la revisión sistemática de Cooper no probó beneficios que apoyen el uso de mifepristone o misoprostol antes de la histeroscopia ambulatoria, reservándose el uso de prostaglandinas vaginales para las mujeres posmenopáusicas cuando el uso de sistemas histeroscópicos es $>5 \mathrm{~mm}$ de diámetro. ${ }^{12}$ Shahid y col. en un estudio doble ciego aleatorio y controlado, evaluaron el nivel de presión de distensión de la cavidad uterina que permitiese una óptima visualización sin incrementar significativamente el dolor, encontrando que la visibilidad era de inferior calidad con $40 \mathrm{~mm}$ $\mathrm{Hg}$ en comparación con presiones entre 70 y $100 \mathrm{~mm}$ $\mathrm{Hg}(\mathrm{p}<0,05)$, mientras que las calificaciones de dolor no difieren en forma significativa con las diferentes 
opciones de presión utilizados en este estudio. ${ }^{29} \mathrm{El}$ Colegio Americano de Ginecólogos y Obstetras recomienda que la presión de distensión de la cavidad uterina debe ser la más baja necesaria para distender la cavidad uterina que permita una adecuada visualización, idealmente por debajo de la presión arterial media. $^{2}$

Camanni y col. compararon el ultrasonido 3D para el cálculo de la puntuación de Lasmar que pronostica la resecabilidad de los miomas submucosos con la $\mathrm{HO}$, encontrando una alta concordancia, aunque el tamaño de muestra fue solo de 34 pacientes. ${ }^{30}$

Utilidad en medicina reproductiva: Guven y col. la emplearon para la evaluación de los leiomiomas intramurales en relación con el éxito de la transferencia embrionaria, encontrando que la tasa de embarazo clínico fue significativamente inferior en el grupo de pacientes con leiomiomas, aunque estos no deformaran la cavidad endometrial y por lo tanto recomiendan la miomectomía en estas pacientes. ${ }^{31}$ Se ha propuesto la $\mathrm{HO}$ antes del inicio de la FIV/ICSI ${ }^{32-34}$ y como parte de los exámenes de primera línea en infértiles ${ }^{35} \sin$ importar la edad, con el objetivo de diagnosticar anomalías intrauterinas menores no vistas con la ecografía transvaginal. ${ }^{34-38}$ La sensibilidad de la histeroscopia diagnóstica es significativamente mayor que la histerosalpingografía (HSG) para la identificación de lesiones intrauterinas no vistas por otros medios diagnósticos, que pueden poner en peligro el éxito de un tratamiento de fertilidad. ${ }^{34}$ En el meta-análisis de El-Toukhy con resultados de cinco estudios mostró beneficios en cuanto a mejor tasa de embarazos en el ciclo de FIV posterior a la $\mathrm{HO}$ ambulatoria ${ }^{39}$, lo cual también fue corroborado en el metaanálisis de Pundir. ${ }^{40}$ Se ha descrito también el uso de este examen para pacientes con más de dos abortos involuntarios, hallándose una alta prevalencia de alteraciones de la cavidad endometrial ${ }^{41}$, y para el diagnóstico y tratamiento de la metaplasia ósea endometrial asociada con infertilidad, considerándose como el estándar de oro para el diagnóstico y tratamiento de esta patología. ${ }^{42}$

Por norma general no debería realizarse histeroscopia en pacientes con estadios avanzados de cáncer en- dometrial por el riesgo de diseminación de células tumorales, dado que podría causar reflujo de células neoplásicas a la cavidad peritoneal, aunque no está claro si esto afecta en forma adversa el pronóstico. ${ }^{2}$

De Angelis en 2003 publicó los resultados de un ensayo clínico aleatorio entre cuyos objetivos estaba validar la presencia de efectos hemodinámicos secundarios en 207 pacientes, cuando se utiliza un minihisteroscopio en el consultorio de $3,3 \mathrm{~mm}$, versus el uso de uno tradicional de $5 \mathrm{~mm}$. Es de anotar que la mayor incidencia de bradicardia fue en el grupo que utilizó óptica de 3,3 mm, no obstante de que fue el de menor dolor y mejor tolerancia al procedimiento.$^{43}$ Estos hallazgos aunque tuvieron significación estadística y contrario a lo esperado por una menor estimulación del ganglio de Frankenauser a nivel del orificio uterino interno que desencadenaría el estímulo vagal (y por lo tanto una bradicardia). Se podría explicar por el uso de curetas de Novak de $4 \mathrm{~mm}$ o por el sistema de distensión con $\mathrm{CO} 2$ que en los equipos de minióptica son de alto flujo, lo cual ocasiona una distensión más rápida y brusca con mayor estimulación en las fibras nerviosas sensibles en la pared uterina, lo cual produciría la activación vasovagal. ${ }^{43}$ En ningún otro de los estudios analizados en esta revisión se describen complicaciones hemodinámicas o arritmias a las que tanto se les teme por parte de los detractores de esta técnica.

\section{Conclusiones}

La histeroscopia con biopsia dirigida es el estándar de oro para la evaluación más exacta de la patología endometrial ${ }^{42}$, en especial cuando se pretende descartar carcinoma endometrial focal. Las biopsias endometriales a ciegas ya no deberían utilizarse como única estrategia de diagnóstico de la HUA en perimenopausia o posmenopausia. ${ }^{1}$ Procedimientos como la histerosonografía, histerosonografía 3D, la biopsia endometrial por aspiración al vacío (Pipelle) y la HO han revolucionado el diagnóstico de la HUA. ${ }^{1,44,45} \mathrm{La}$ $\mathrm{HO}$ es considerada como un procedimiento mínimamente invasivo y con una adecuada técnica es aceptada y bien tolerada por la mayoría de las pacientes ${ }^{15}$, incluso sin requerir anestesia ${ }^{46}$, tal como lo demostró en su estudio Filiz y col. quienes la compararon con 
la histeroscopia convencional de quirófano, donde las pacientes informaron mejor nivel de satisfacción con la técnica oficinal y un menor costo. ${ }^{15}$ En cuanto al uso de misoprostol para la preparación cervical antes del procedimiento, algunos estudios han mostrado resultados contradictorios $^{10,12-14}$, mientras otros con mejor nivel de evidencia concluyen que no se debería recomendar la administración sistemática de misoprostol antes de la histeroscopia ambulatoria, y que la preparación cervical con prostaglandinas vaginales debería considerarse para mujeres posmenopáusicas cuando se utilicen sistemas histeroscópicos mayores de $5 \mathrm{~mm}$ de diámetro. ${ }^{12}$

\section{Referencias}

1. Kotdawala P, Kotdawala S, Nagar N. Evaluation of endometrium in peri-menopausal abnormal uterine bleeding. J Midlife Health. 2013; 4(1):16-21.

2. American College of Obstetricians and Gynecologists. Technology assessment No. 7: Hysteroscopy. Obstet Gynecol. 2011; 117(6):1486-91.

3. Munro MG, Critchley HO, Broder MS, Fraser IS, FIGO Working Group on Menstrual Disorders. FIGO classification system (PALM-COEIN) for causes of abnormal uterine bleeding in nongravid women of reproductive age. Int J Gynaecol Obstet. 2011; 113(1):3-13.

4. Valle RF. Development of hysteroscopy: from a dream to a reality, and its linkage to the present and future. J Minim Invasive Gynecol. 2007; 14(4):407-18.

5. Bettocchi S, Nappi L, Ceci O, Selvaggi L. Office hysteroscopy. Obstet Gynecol Clin North Am. 2004; 31(3):641-54, xi.

6. Emanuel MH. New developments in hysteroscopy. Best Pract Res Clin Obstet Gynaecol. 2013;27(3):421-9.

7. Cooper NA, Smith P, Khan KS, Clark TJ. A systematic review of the effect of the distension medium on pain during outpatient hysteroscopy. Fertil Steril. 2011, 95(1):264-71

8. Craciunas L, Sajid MS, Howell R. Carbon dioxide versus normal saline as distension medium for diagnostic hysteroscopy: a systematic review and meta-analysis of randomized controlled trials. Fertil Steril. 2013; 100(6):1709-14.e1-4.

9. Munro MG, Storz K, Abbott JA, Falcone T, Jacobs VR, Muzii L, et al. AAGL Practice Report: Practice Guidelines for the Management of Hysteroscopic Distending Media: (Replaces Hysteroscopic Fluid Monitoring Guidelines. J Minim Invasive Gynecol. 2013; 20(2):137-48.

10. Bastu E, Celik C, Nehir A, Dogan M, Yuksel B, Ergun B. Cervical priming before diagnostic operative hysteroscopy in infertile women: a randomized, double-blind, controlled comparison of 2 vaginal misoprostol doses. Int Surg. 2013; 98(2):140-4

11. Ben-Chetrit A, Eldar-Geva T, Lindenberg T, Farhat M, Shimonovitz S, Zacut D, et al. Mifepristone does not induce cervical softening in non-pregnant women. Hum Reprod. 2004; 19(10):2372-6.

12. Cooper NA, Smith P, Khan KS, Clark TJ. Does cervical preparation before outpatient hysteroscopy reduce women's pain experience? A systematic review. BJOG. 2011; 118(11):1292-301.

13. Hassa H, Aydin Y, Oge T, Cicek K. Effectiveness of vaginal misoprostol and rectal nonsteroidal anti-inflammatory drug in vaginoscopic diagnostic outpatient hysteroscopy in primarily infertile women: double-blind, randomized, controlled trial. J Minim Invasive Gynecol. 2013; 20(6):880-5

14. Esin S, Baser E, Okuyan E, Kucukozkan T. Comparison of sublingual misoprostol with lidocaine spray for pain relief in office hysteroscopy: a randomized, double-blind, placebo-controlled trial. J Minim Invasive Gynecol. 2013; 20(4):499-504
15. Filiz T, Doğer E, Corakçı A, Ozeren S, Calışkan E. The efficacy, cost and patient satisfaction of classic versus office hysteroscopy in cases with suspected intrauterine space occupying lesions with 3-dimension ultrasound and abnormal uterine bleeding. J Turk Ger Gynecol Assoc. 2009; 10(4):189-93.

16. van Kerkvoorde TC, Veersema S, Timmermans A. Long-term complications of office hysteroscopy: analysis of 1028 cases. J Minim Invasive Gynecol. 2012; 19(4):494-7.

17. van Dongen H, Timmermans A, Jacobi CE, Elskamp T, de Kroon CD, Jansen FW. Diagnostic hysteroscopy and saline infusion sonography in the diagnosis of intrauterine abnormalities: an assessment of patient preference. Gynecol Surg. $2011 ; 8(1): 65-70$

18. Guida M, Di Spiezio Sardo A, Acunzo G, Sparice S, Bramante S, Piccoli R, et al. Vaginoscopic versus traditional office hysteroscopy: a randomized controlled study. Hum Reprod. 2006; 21(12):3253-7.

19. McMartin K. Hysteroscopic tubal sterilization: an evidence-based analysis. Ont Health Technol Assess Ser. 2013; 13(21):1-35

20. Kasius JC, Broekmans FJ, Fauser BC, Devroey P, Fatemi HM. Antibiotic prophylaxis for hysteroscopy evaluation of the uterine cavity. Fertil Steril. 2011; 95(2):792-4.

21. Gregoriou O, Bakas P, Grigoriadis C, Creatsa M, Sofoudis C, Creatsas G. Antibiotic prophylaxis in diagnostic hysteroscopy: is it necessary or not? Eur J Obstet Gynecol Reprod Biol. 2012; 163(2):190-2

22. Alanís Fuentes J, Amoroso Hernández MA. [Of office hysteroscopy for the removal of intrauterine device. Literature review]. Ginecol Obstet Mex. 2009; 77(4):197-201.

23. Fonseca MD, Sessa FV, Resende JA, Guerra CG, Andrade CM, Crispi CP. Identifying predictors of unacceptable pain at office hysteroscopy. J Minim Invasive Gynecol. 2014 Jul-Aug; 21(4):586-91.

24. Sharma JB, Aruna J, Kumar P, Roy KK, Malhotra N, Kumar S. Comparison of efficacy of oral drotaverine plus mefenamic acid with paracervical block and with intravenous sedation for pain relief during hysteroscopy and endometrial biopsy. Indian J Med Sci. 2009; 63(6):244-52.

25. Munro MG, Brooks PG. Use of local anesthesia for office diagnostic and operative hysteroscopy. J Minim Invasive Gynecol. 2010; 17(6):709-18.

26. Van den Bosch T, Van Schoubroeck D, Daemen A, Domali E, Vandenbroucke $\mathrm{V}$, De Moor B, et al. Lidocaine does not reduce pain perception during gel instillation sonography or subsequent office hysteroscopy: results of a randomized trial. Gynecol Obstet Invest. 2011; 71(4):236-9.

27. Van den Bosch T, Betsas G, Van Schoubroeck D, Daemen A, Vandenbroucke V, Cornelis A, et al. Gel infusion sonography in the evaluation of the uterine cavity. Ultrasound Obstet Gynecol. 2009; 34(6):711-4.

28. Ahmad G, Attarbashi S, O'Flynn H, Watson AJ. Pain relief in office gynaecology: a systematic review and meta-analysis. Eur J Obstet Gynecol Reprod Biol. 2011; 155(1):3-13

29. Shahid A, Pathak M, Gulumser C, Parker S, Palmer E, Saridogan E. Optimum uterine filling pressure for outpatient diagnostic hysteroscopy: a double-blind, randomized controlled trial. Reprod Biomed Online. 2014; 28(1):86-91.

30. Camanni M, Bonino L, Tessarolo M, Migliaretti G, Ferrero B, Deltetto F. Is it possible to obtain a presurgical Lasmar score for hysteroscopic myomectomy by ultrasound alone? Ultrasound Obstet Gynecol. 2012; 40(1):106-11.

31. Guven S, Kart C, Unsal MA, Odaci E. Intramural leoimyoma without endometrial cavity distortion may negatively affect the ICSI - ET outcome. Reprod Biol Endocrinol. 2013; 11: 102

32. Karayalçin R, Ozyer S, Ozcan S, Uzunlar O, Gürlek B, Moraloğlu O, et al. Office hysteroscopy improves pregnancy rates following IVF. Reprod Biomed Online. 2012; 25(3):261-6.

33. Kasius JC, Eijkemans RJ, Mol BW, Fauser BC, Fatemi HM, Broekmans FJ. Cost-effectiveness of hysteroscopy screening for infertile women. Reprod Biomed Online. 2013; 26(6):619-26.

34. Bakas P, Hassiakos D, Grigoriadis C, Vlahos N, Liapis A, Gregoriou O. Role of hysteroscopy prior to assisted reproduction techniques. J Minim Invasive Gynecol. 2014; 21(2):233-7. 
35. Pansky M, Feingold M, Sagi R, Herman A, Schneider D, Halperin R. Diagnostic hysteroscopy as a primary tool in a basic infertility workup. JSLS. 2006; 10(2):231-5.

36. Fatemi HM, Kasius JC, Timmermans A, van Disseldorp J, Fauser BC, Devroey $\mathrm{P}$, et al. Prevalence of unsuspected uterine cavity abnormalities diagnosed by office hysteroscopy prior to in vitro fertilization. Hum Reprod. 2010;25(8):195965.

37. Smit JG, Kasius JC, Eijkemans MJ, Koks CA, Van Golde R, Oosterhuis JG et al. The inSIGHT study: costs and effects of routine hysteroscopy prior to a first IVF treatment cycle. A randomised controlled trial. BMC Womens Health. 2012;12:22

38. Koskas M, Mergui JL, Yazbeck C, Uzan S, Nizard J. Office hysteroscopy for infertility: a series of 557 consecutive cases. Obstet Gynecol Int. 2010; 2010: 168096.

39. El-Toukhy T, Sunkara SK, Coomarasamy A, Grace J, Khalaf Y. Outpatient hysteroscopy and subsequent IVF cycle outcome: a systematic review and metaanalysis. Reprod Biomed Online. 2008; 16(5):712-9.

40. Pundir J, Pundir V, Omanwa K, Khalaf Y, El-Toukhy T. Hysteroscopy prior to the first IVF cycle: a systematic review and meta-analysis. Reprod Biomed Online. 2014; 28(2):151-61.
41. Souza CA, Schmitz C, Genro VK, Martins A, Scheffel C, Oppermann ML, et al. Office hysteroscopy study in consecutive miscarriage patients. Rev Assoc Med Bras. 2011;57(4):397-401.

42. Rosa-E-Silva JC, Barcelos ID, Navarro PA, Rosa-E-Silva AC, Nogueira AA, Ferriani RA. Osseous metaplasia of the endometrium associated with infertility: a case report and review of the literature. J Med Case Rep. 2009; 3:7427.

43. De Angelis C, Santoro G, Re ME, Nofroni I. Office hysteroscopy and compliance: mini-hysteroscopy versus traditional hysteroscopy in a randomized trial. Hum Reprod. 2003; 18(11):2441-5.

44. Ngu SF, Cheung VY, Pun TC. Randomized study of vaginoscopy and H Pipelle vs traditional hysteroscopy and standard Pipelle. J Minim Invasive Gynecol. 2012; 19(2):206-11.

45. Negm SM, Kamel RA, Abuhamila FA. Three-dimensional sonohysterography compared with vaginoscopic hysteroscopy for evaluation of the uterine cavity in patients with recurrent implantation failure in in vitro fertilization cycles. $\mathrm{J}$ Minim Invasive Gynecol. 2012; 19(4):503-8.

46. Cicinelli E. Hysteroscopy without anesthesia: review of recent literature. J Minim Invasive Gynecol. 2010; 17(6):703-8. 